\title{
Survey on Fingerprint Enhancement Techniques
}

\author{
M.Manju \\ Assistant Professor, Department of CSE, \\ Jayamatha Engineering College, \\ Aralvaimozhi, India
}

\author{
V.Kavitha, PhD. \\ Associate Professor, Department of CSE, \\ University College of Engineering,Nagercoil, \\ Anna University Tirunelveli, India.
}

\begin{abstract}
In the field of security, many Automatic Fingerprint Identification System (AFIS) have been developed. One of the indices for evaluating the security of such system is the quality of the fingerprint image and the efficiency of the enhancement algorithm used. The main aim of the paper is to present a survey on traditional and classical fingerprint image enhancement techniques. The performance of the enhancement algorithms are evaluated on the basis of PSNR and MSE values. Based on the above measures, the most efficient enhancement algorithm is chosen.
\end{abstract}

\section{Keywords:}

AFIS, HE, LPD, directional filters, Gabor filters, MSE, PSNR.

\section{INTRODUCTION}

Image enhancement is a pre-processing step which is used to improve the quality of the image by removing blur and noise. Among the security variables [key, password, fingerprint], fingerprint is the most commonly and widely used entity since they are unique and do not change over time and moreover they never need to be remembered.

Fingerprint images are direction-oriented patterns formed by ridges and valleys. These images are rarely of good quality. They are degrades and corrupted due to noise and other factors like variations in skin, impression conditions etc. Hence fingerprint image enhancement is the first step in any Automatic Fingerprint Identification System (AFIS), which improves the quality of the fingerprint image thereby increasing the reliability of fingerprint recognition.

The ingredients of fingerprint that are used in any AFIS are global features (or) local features. The global features include ridge orientation map, ridge frequency map and singular points and the minutiae points constitute the local features.

Ridge orientation map - They are the local direction of the ridge-valley structure. They are used for classification purposes, enhancement feature verification and filtering.

Ridge Frequency map - They are the reciprocal of the ridge distance in the direction perpendicular to the local ridge orientation. They are used for filtering of fingerprint images.
Singular points - They are the discontinuities in the orientation field. They are used for fingerprint registration and classification. There are two types:

i) Core - It is the uppermost part of a curving ridge.

ii) Delta - It is a point where three ridge flows meet.

Minutiae - They are the local discontinuities of the ridge structure. Among the minutiae types, ridge endings and bifurcation are the most commonly used fingerprint representation in all AFIS.

Many enhancement algorithms have been proposed based on the above said features.

This paper is organized as follows: Part 2 deals with the various fingerprint image enhancement techniques. Part 3 deals with the comparison of the enhancement techniques and finally a brief conclusion section is given which summarizes the paper.

\section{FINGERPRINT ENHANCEMENT TECHNIQUES}

The various fingerprint enhancement techniques are discussed below:

\subsection{Enhancement using Histogram Equalization (HE):}

It is a contrast enhancement technique which distributes the pixel values uniformly such that the enhanced image has linear cumulative histogram [1]. Histogram equalization is a technique for adjusting image intensities to enhance contrast [3]. The main concept behind this technique is to re-assign the intensity values of pixels to make the intensity distribution uniform. $\mathrm{HE}$ is considered to be a global operation. It stretches the histogram across the entire spectrum of pixels ( 0 $255)$. It can be used to obtain a new image by the process that, for each brightness level ' $\mathrm{j}$ ' in the original images the new pixel value ' $\mathrm{k}$ ' is calculated as shown in equation 1 .

$$
k=\sum_{i=n}^{j} \frac{N_{i}}{T}
$$


where the sum $\sum$ counts the number of pixels in the image with brightness equal to (or) less than ' $\mathrm{j}$ ' and $\mathrm{T}$ is the total number of pixels.

The main aim of HE is to find a gray-level transformation function $\mathrm{T}$ to transform an image ' $\mathrm{f}$ ' such that the histogram of $T$ (f) is equalized.

The main advantage of $\mathrm{HE}$ is that it is very simple and efficient.

The drawbacks are, they tend to change the brightness of the image and they do not preserve the brightness of the image.

\subsection{Enhancement using 2D Fourier Transform} (FT):

Fourier Transform is a transformation technique that transforms the image from spatial domain to frequency domain. The steps involved in enhancing the fingerprint using this transform are given in Figure 1.

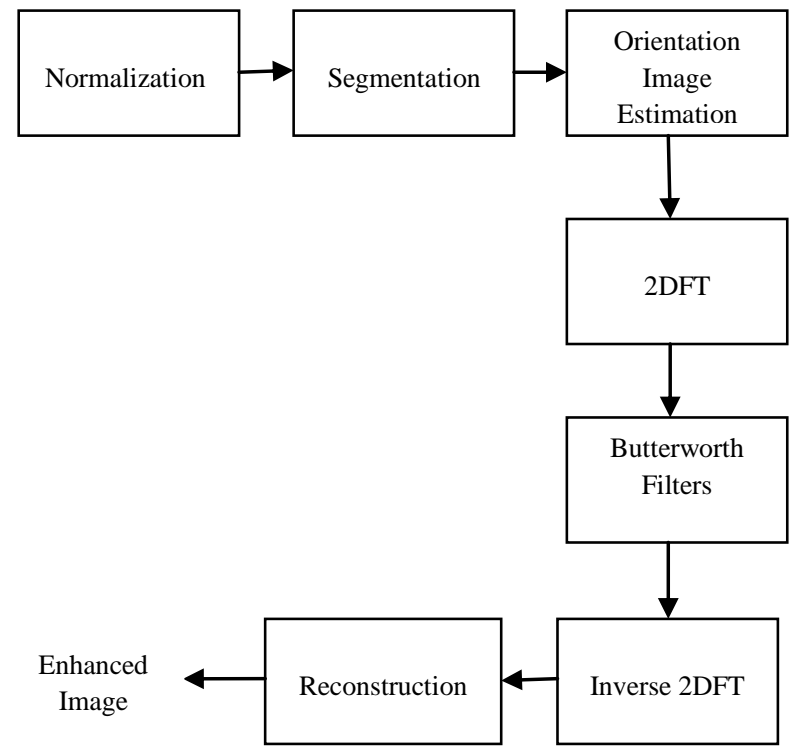

Figure. 1 Steps involved in Enhancement using 2DFT

Normalization: It is done to reduce the differences in grey level values along the ridges and valleys so that the pixel values in the image have a specified mean and variance. The desired mean and variance were chosen to be 0 and 1 respectively, so that the new intensities of the pixels for the normalized image would be between -1 and 1 .

Segmentation: It is done to separate the actual fingerprint area from the image background by dividing the image into many blocks. A threshold is set to exclude the background from the fingerprint area. The

Orientation image Estimation: Orientation image is a representation of the directions of the ridges and valleys in a fingerprint. Orientation image estimation is used to estimate the orientation of the image by placing an image window at a point in the raw image and the window is rotated in sixteen equally spaced directions and the projections are calculated along the $y$ direction.[3]The projection with maximum variance is fixed as the orientation of the pixel.

2DFT: By using 2D Fourier Transform the image is converted from spatial domain to frequency domain.

Butterworth Filters: The image in frequency domain is then filtered through 16 Butterworth filters with each filter tuned to a particular orientation. The number of directional filters corresponds to the set of directions used to calculate the orientation image.

Inverse 2DFT: After each directional has been applied to the frequency domain image, the inverse Fourier transform is used to convert each image back to the spatial domain, producing a set of directionally filtered image called as prefiltered images.

Reconstruction: The final stage of the enhancement process is to reconstruct the image from the pre-filtered image by using the value of the ridge orientation at each pixel in the raw image and the filtering direction of each pre-filtered image.

\section{Disadvantage:}

i. The frequency content was taken to be constant throughout.

\subsection{Enhancement using Gabor filters:}

It is an enhanced version of the previous method in which the frequency content of the image is taken into account [2]. The steps involved in this method are illustrated below in Figure. 2

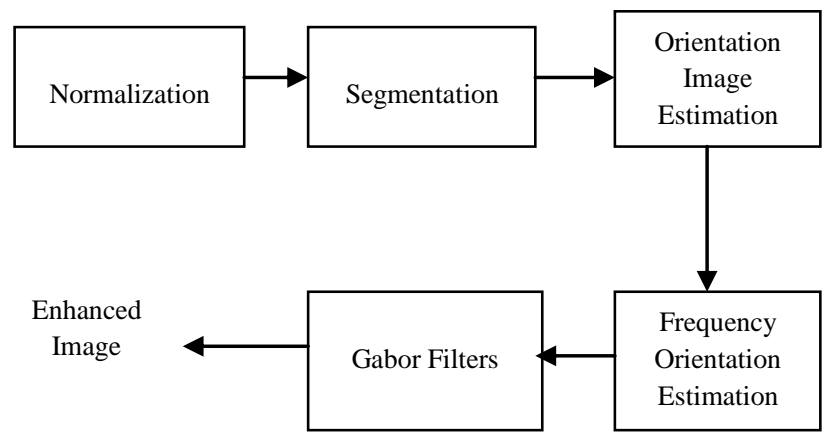

Figure. 2 Steps involved in Enhancement using Gabor Filters

Normalization: As discussed in Section 2.2.

Segmentation: As explained in Section 2.2.

Orientation Image Estimation:

1. The normalized image is divided into blocks of $8 \mathrm{x}$ 8 pixels.

2. The gradient of each pixel is computed using the sobel operator which is given below: 


\begin{tabular}{|c|c|c|}
\hline-1 & -2 & -1 \\
\hline 0 & 0 & 0 \\
\hline 1 & 2 & 1 \\
\hline
\end{tabular}

\begin{tabular}{|l|l|l|}
\hline-1 & 0 & -1 \\
\hline-2 & 0 & -2 \\
\hline-1 & 0 & -1 \\
\hline
\end{tabular}

3. The above given masks are convolved with the normalized image to get the gradients in the $\mathrm{x}$ and $\mathrm{y}$ directions.

4. The moments are then calculated for each block using the expressions given below:

$$
\begin{aligned}
& V_{x}(i, j)= \\
& \sum_{u=i-w / 2}^{i+w / 2} \sum_{v=i-w / 2}^{i+w / 2} 2 \partial_{\mathrm{x}}(\mathrm{u}, \mathrm{v}) \partial_{\mathrm{y}}(\mathrm{u}, \mathrm{v})--(2)
\end{aligned}
$$

$$
\begin{aligned}
& V_{y}(i, j)= \\
& \sum_{u=i-w / 2}^{i+w / 2} \sum_{v=i-w / 2}^{i+w / 2} \partial_{\mathrm{x}}{ }^{2}(\mathrm{u}, \mathrm{v}) \partial_{\mathrm{y}}{ }^{2}(\mathrm{u}, \mathrm{v})---(3)
\end{aligned}
$$

Where $V_{x}(i, j)$ and $V_{y}(i, j)$ are the moments in $\mathrm{x}$ and $\mathrm{y}$ direction respectively, $\partial_{\mathrm{x}}$ and $\partial_{\mathrm{y}}$ are the $\mathrm{x}$ and $\mathrm{y}$ gradients and $w$ is the block size taken as 8 .

5. The orientation of each block is computed using the following expression:

$$
\theta(i, j)=\frac{1}{2} \tan ^{-1} \frac{V_{y}(i, j)}{V_{x}(i, j)}
$$

Frequency Orientation Estimation: It estimates the approximate ridge frequency for the fingerprint image by dividing it into blocks of $8 \times 8$ pixels.

Gabor Filtering: Finally filtering is performed to remove noise and preserve the ridge structures. The image is filtered by a bank of Gabor filters (Band Pass Filters) tuned to the corresponding local frequency and orientation for each neighborhood. The general form of Gabor filter is given below:

$$
\begin{gathered}
h(x, y, \emptyset, f)=\exp \left(\left(-\left[\frac{\mathrm{x}_{\phi^{2}}}{\partial_{\mathrm{x}^{2}}}+\frac{\mathrm{y}_{\phi^{2}}}{\partial_{\mathrm{y}^{2}}}\right]\right) \cos \left(2 \pi \mathrm{fx}_{\emptyset}\right)-(5)\right. \\
\text { here, } \mathrm{x}_{\emptyset}=\mathrm{x} \cos \emptyset+\mathrm{y} \sin \emptyset \text { and }-\mathrm{y}_{\emptyset}=-\mathrm{x} \sin \emptyset+\mathrm{y} \cos \emptyset \\
\mathrm{y}_{\varnothing}
\end{gathered}
$$

Where $\partial_{\mathrm{x}}$ and $\partial_{\mathrm{y}}$ are the standard deviations of the Gaussian envelope along the $\mathrm{x}$ and $\mathrm{y}$ axes respectively, $\emptyset$ is the orientation of the filter and $\mathrm{f}$ is the frequency of the sinusoidal wave.

\section{Advantages:}

i. The enhanced image is of high efficiency since the Gabor filter has both frequency selective and orientation selective properties.

ii. The method have optimal resolution in both spatial and frequency domains.

\section{Disadvantages:}

i. Reduction in contrast.

ii. Produces a large number of spurious minutiae points.

iii. High computational complexity.

iv. Overall increase in running time of identification and verification process.

v. Creation of artifacts in the enhanced image.

vi. Increase in identification and verification errors.

\subsection{Enhancement using Gabor filters combined with wavelet transforms:}

Wavelet transform is an effective tool in reducing noise. Images are analyzed at multiple scales [6]. Wavelet coefficients contain both time and frequency information as basis functions. This method is illustrated in Figure 3.

\section{Normalization: As discussed in Section 2.2.}

Wavelet Decomposition: The image is decomposed into multi-resolution representation using wavelet transform. The convolution of the different base function with the input signal causes different effects in different resolutions. Generally one or two decomposition levels are selected. A discrete wavelet transform can be used to compute the multiresolution representation of signals which is shown in Figure 4. The approximation $c\left(A_{j+1}\right)$ can be obtained by convolving $A_{j+1}$ with the low pass filter. The detail $c\left(D_{j+1}\right)$ is computed by convolving $c\left(\mathrm{~A}_{j}\right)$ with high pass filter.

Wavelet Coefficient Adjustment: After decomposition, a series of wavelet coefficients are obtained, each of which corresponds to resolution level on the dyadic scale.

Gabor Filtering: As explained in Section 2.3.

Reconstruction: After modifying the approximation sub-mage with Gabor filters, the final image of the enhancement process is reconstructed. Normally the wavelets used in reconstruction are the same as that used in the decomposition process.

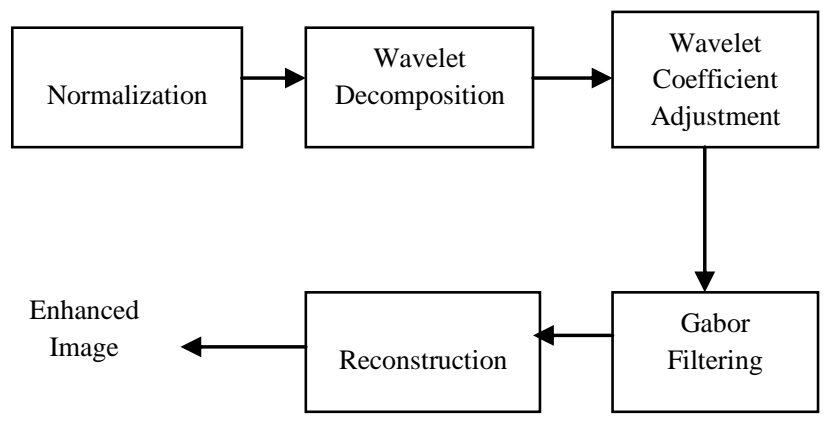

Figure. 3 Steps involved in Enhancement using Gabor Filters combined with wavelet transform 
Advantages:

i. Effective de-noising.

ii. Increases the contrast between the ridge and the background.

iii. Improved enhanced image.

\section{Disadvantage:}

i. Computationally expensive.

\subsection{Enhancement using Directional Filters:}

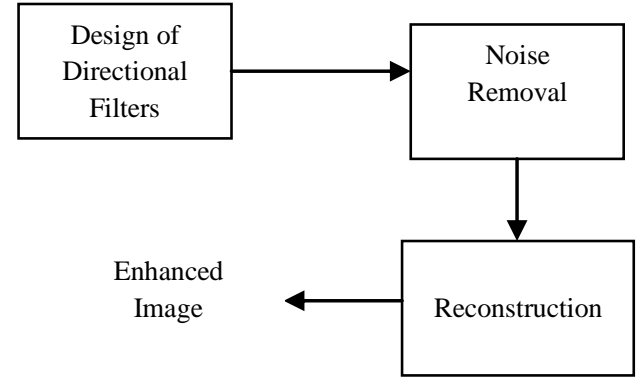

Figure 4: Steps involved in Enhancement using Directional Filters.

Design of Directional Filters: In DFB (Directional Filter Bank), the fingerprint image is divided into eight parts according to the orientation of the ridge structure, which is given in Figure 5.

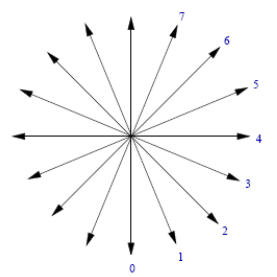

Figure 5: Orientation of Fingerprint Ridge Flow.

Directional filtering can be implemented with fan type band pass filters as shown in Figure 6. A directional filter is a collection of digital filters with common input or output [4].
This technique is commonly used in multi-resolution enhancement process. It works by decomposing the fingerprint image into different frequency bands or sub images. In fingerprint preprocessing, the standard median filters with rectangular topology are difficult in reducing noise and hence directional filters are used [6]. The process involved in this technique is given in Fig.4.
Analysis

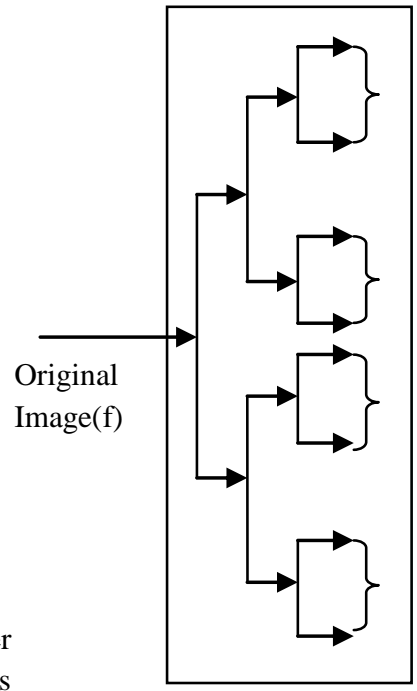

Synthesis

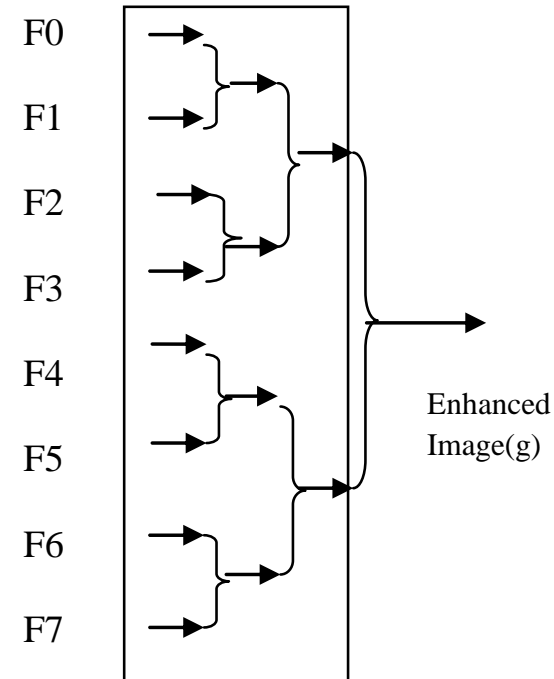

Figure 6: Analysis and Synthesis process in DFB

The directional filter consists of an analysis bank and a synthesis bank. The analysis bank splits the original image into eight directionally passed sub band images while the synthesis bank combines the sub band images into one image. The directional image can be regarded as decomposition of the original image in eight pieces based on direction. Directional image contain features, associated with global direction rather than local directions.

Noise Removal: It is accomplished by calculating the block based directional energy of each directional image. The directional energy of a block $(X, Y)$ including the pixel $(x, y)$ from the $\mathrm{K}_{\mathrm{th}}$ directional image is calculated as follows:

$$
E_{K}(X, Y)=\sum_{x=0}^{m_{k}} \sum_{y=0}^{n_{k}}\left|f_{K}(X, Y ; x, y)\right|
$$

Where, $\mathrm{f}_{\mathrm{k}}$ is the directional image.

The noise free directional images represented by $A_{k}$ are obtain by the equation:

$$
A_{K}=\left\{\begin{array}{l}
f_{k}(X, Y ; x, y) \text { if } E_{k}(X, Y)>T \\
f_{k}(X, Y ; x, y) \text { if otherwise }
\end{array}\right.
$$

Here, T represents the threshold.

Reconstruction: The enhanced image is constructed form the directional image, according to the following equation:

$f_{h f}(X, Y)=\max _{1 \leq i \leq 8} f_{i}(X, Y)$ 


\begin{tabular}{|cc|}
\hline \multicolumn{1}{|c|}{ a) } & Decomposition \\
\hline Gaussian-like & Laplacian-like \\
\hline $\mathrm{g}_{1}=\operatorname{reduce}\left(f_{p}, f_{0}\right) ;$ & $l_{1}=g_{1}-\operatorname{expand}\left(g_{2}, f\right) ;$ \\
$\mathrm{g}_{2}=\operatorname{reduce}\left(g_{1}, f\right) ;$ & $l_{2}=g_{2}-\operatorname{expand}\left(g_{3}, f\right) ;$ \\
$\mathrm{g}_{3}=\operatorname{reduce}\left(g_{2}, f\right) ;$ & $l_{3}=g_{3}$-expand $\left(g_{4}, f\right) ;$ \\
$\mathrm{g}_{4}=\operatorname{reduce}\left(g_{3}, f\right) ;$ & \\
\hline & b) $\operatorname{Reconstruction}$ \\
\hline & $f_{\mathrm{p}}=\operatorname{expand}\left(., f_{o}\right)$ \\
& $\operatorname{expand}(., f)+l_{1}$ \\
& $\operatorname{expand}\left(l_{3}, f\right)+l_{2}$ \\
\hline
\end{tabular}

Where, $\mathrm{f}_{\text {hf }}$ is high frequency output image from directional filter bank and fi represent $i^{\text {th }}$ directional image. For every block $(\mathrm{X}, \mathrm{Y})$ of the original image, a replacement is selected, from the eight directional image base, on maximum directional energy.

\section{Advantages:}

i. This method is effective in both dry fingerprint images and wet fingerprint images.

ii. It removes noise in between the ridge and filling the white hole in the ridge.

iii. This leads to decrease the False Acceptance Rate (FAR) and False Rejection Rate (FRR), in fingerprint minutiae extraction.

\subsection{Enhancement using LPD:}

Fingerprint recognition system depends on the quality of the input image. To improve the quality of the image and to increase the recognition rate and to ensure low error rate, the fingerprint is first subjected to enhancement technique. This paper has incorporated Laplacian-based pyramidal decomposition for enhancement which is illustrated in Figure 7.

\section{Pyramid Decomposition (PD)}

Pyramid decomposition [4] requires resizing (scaling, or geometric transformation). In order to create our Gaussian and Laplacian- like pyramids, it is defined to reduce $(I, f)$ and expand $(I, f)$ operations, which decrease and increase an image $I$ in size by the factor $f$, respectively. On reducing, the image is initially low-pass filtered to prevent aliasing using a Gaussian kernel. The latter's standard deviation depends on the resizing factor, which here follows the lower bound approximation of the corresponding ideal low-pass filter, $\sigma=0.75 \cdot f / 2$ [3]. Initially the original fingerprint image $f p$ is reduced by a factor of $f_{\mathrm{o}} \geq 1.5$ in order to exclude the highest frequencies. In further step the image size is reduced by a factor $f \leq 1.5$ for three times. To create images containing only band limited signals of the original image, one of the three original images is expanded, $g_{2-4}$ by factor $f$ and subtract each of them from the next lower level, yielding $l_{1-3}$.[5]. The entire process is tabulated as shown in Table 1.

\section{Orientation Estimation (OE)}

The next step is calculation of orientation of the image. Orientation calculation is critical for fingerprint image enhancement in both frequency and spatial domain. The orientation image is the local orientation of the ridges and is represented as a matrix of direction vectors. Most of the fingerprint classification and identification processes calculate the local ridge orientation of the fixed-block size instead of each pixel.

Table 1. Pyramidal Decomposition (PD)

Before determining the orientation of the fingerprint, it is subjected to three levels of filtering:
- De-noising.
- Inverse Filtering.
- Wiener Filtering.

Fast Fourier Transform (FFT) of the original image is convoluted to remove white noise followed by the removal of additive noise by inverse filtering. The inverse filtering is a restoration technique for de-convolution, i.e., when the image is blurred by a known low pass filter, it is possible to recover the image by inverse filtering or generalized inverse filtering. However, inverse filtering is very sensitive to additive noise. The wiener filtering executes an optimal tradeoff between inverse filtering and noise smoothing. It removes the additive noise and inverts the blurring simultaneously. The wiener filtering is optimal in terms of the mean square error. In other words, it minimizes the overall mean square error in the process of inverse filtering and noise smoothing. The wiener filtering is a linear estimation of the original image.

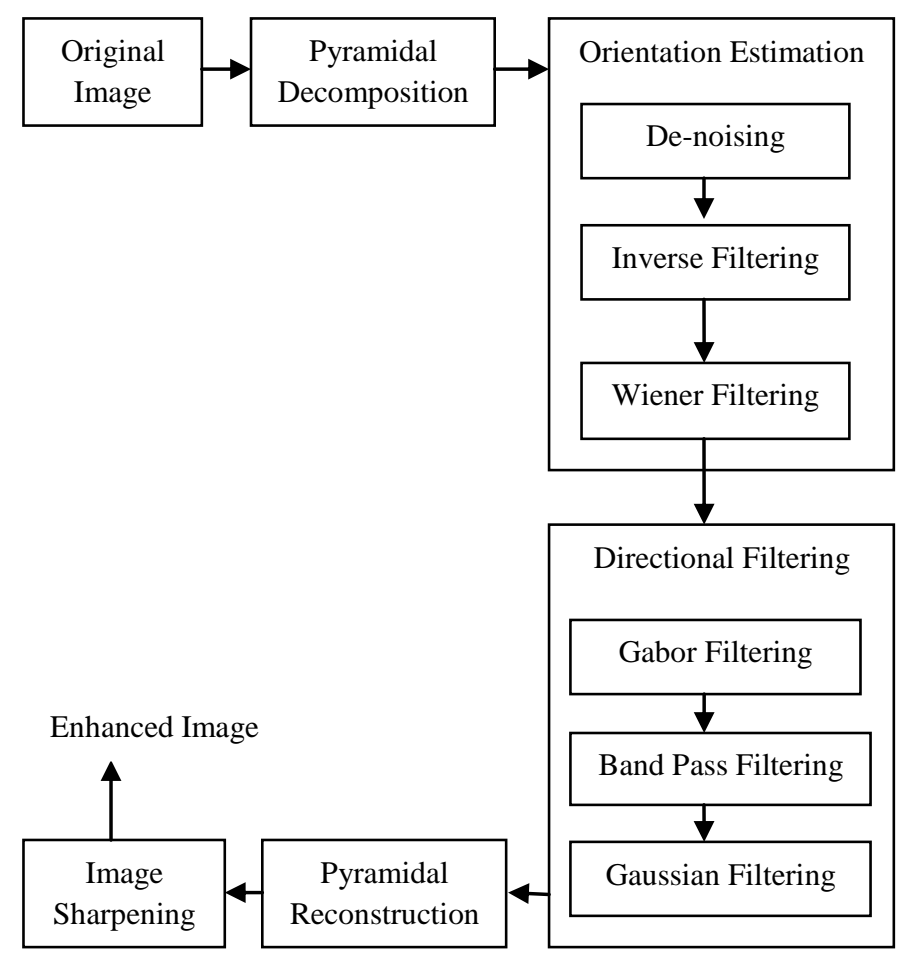

Figure 7: Steps involved in Laplacian-based Pyramidal Decomposition 


\section{Directional Filter (DF)}

To enhance the SNR (signal-to-noise ratio), i.e., to remove sweat pores, scars, etc., the directional averaging to all levels $l 1-3$ is applied independently. The local filtering direction within $l i$ is given by $\angle(\mathrm{LSi}) / 2-\pi / 2$, thus it follows the ridges/valleys of the fingerprint. At every point, the neighboring pixels along a line having the same local direction are averaged with a (1-D) Gaussian, yielding the new value. The possible number of different averaging directions is quantized

\section{Advantages:}

i. It does not suffer from any artifacts

ii. It provides higher accuracy for fingerprint recognition.

\section{COMPARISON AND DISCUSSION}

The main aim of fingerprint image enhancement is to produce a good visual appearance of the image and also improve feature detection like ridge detection and minutiae extraction thereby avoiding undesired side effects in the subsequent processing. The performance measures that are used for the comparative study are given as follows:

\section{a) PSNR: Peak Signal-to-noise ratio}

The PSNR computes the peak signal-to-noise ratio and represents a measure of the peak error in decibels, between two images. This ratio is often used as a quality measurement between the original and a compressed or reconstructed image. PSNR is expressed as follows:

$$
\text { PSNR }=10 \log _{10}\left(\frac{R * R}{M S E}\right)
$$

MSE in denominator represents the cumulative squared error between the reconstructed and the original image. The lower value of MSE represents the lower error in the reconstruction of the image. The mean-squared error is expressed as follows:

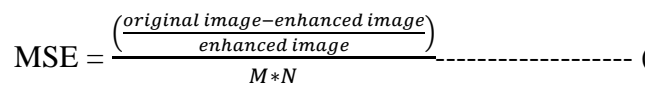

where $\mathrm{M}, \mathrm{N}$ represents the rows and columns of Image.

\section{b) EER: Equal Error rate}

It refers to robustness and stability of algorithm. At this value both the false acceptance rate values and false rejection rate values are equal. The False Acceptance Rate (FAR) is the success probability for an unauthorized user or a user that does not exist within a biometric system to be falsely recognized as the legally registered user. A low tolerance threshold for the biometric data to be matched leads to a lower FAR value, but to higher values of the False Rejection Rate (FRR). In contrast, the False Rejection Rate (FRR) rate is the probability of the legally registered user to be falsely rejected by the biometric system when presenting his biometric feature. High tolerance limits for the biometric data to match lead to a very low FRR value, but to higher values for the False Acceptance Rate (FAR). Both values FAR and FRR are negatively correlated. However, these measures can vary significantly depending on how one adjusts the sensitivity of the mechanism that matches the biometric. The lower the equal error rate is, the higher the accuracy is.

\section{c) Computation Time:}

This metric is used to estimate the processing time involved in enhancing the image. This is observed by using CPU time command of Mat lab.

Table 2: Comparison of the discussed approaches

\begin{tabular}{|l|l|l|c|}
\hline $\begin{array}{l}\text { Enhancement } \\
\text { Technique }\end{array}$ & EER & PSNR & $\begin{array}{c}\text { Computation } \\
\text { Time }\end{array}$ \\
\hline $\begin{array}{l}\text { Histogram } \\
\text { Equalization }\end{array}$ & $11.2 \%$ & $31.036 \mathrm{db}$ & $0.253 \mathrm{secs}$ \\
\hline FT based & $10.978 \%$ & $35.67 \mathrm{db}$ & $0.78 \mathrm{secs}$ \\
\hline Gabor Filters & $9.8 \%$ & $43.32 \mathrm{db}$ & $0.928 \mathrm{secs}$ \\
\hline $\begin{array}{l}\text { Directional } \\
\text { Filters }\end{array}$ & $9.89 \%$ & $38.24 \mathrm{db}$ & $0.534 \mathrm{secs}$ \\
\hline $\begin{array}{l}\text { Gabor filter in } \\
\text { Wavelet Domain }\end{array}$ & $9.976 \%$ & $39.45 \mathrm{db}$ & $0.707 \mathrm{secs}$ \\
\hline LPD & $9.345 \%$ & $41.56 \mathrm{db}$ & $0.894 \mathrm{secs}$ \\
\hline
\end{tabular}

\section{CONCLUSION}

Different enhancement approaches have shown to improve the recognition performance in different studies. It is observed that the LPD method and Wavelet based enhancement is giving better results. The fact is supported from the above qualitative observations given above, supported experimentally by using publicly available databases and standard minutiae matching techniques. It can be observed that there is tradeoff between the CPU times and Peak signal to noise ratio .Higher values of PSNR is expected for good recognition rate which also reduces the equal error rate.

\section{REFERENCES}

[1]. Anto Melvin Paul, R.Mary Lourde, "A Study on Fingerprint Enhancement Techniques for Fingerprint Identification", Proceedings of IEEE International Conference on Video and Signal Based Survillence, 2006.

[2]. T.Vidhya, T.K.Thivakaran, "Fingerprint Image Enhancement using Wavelet over Gabor Filters", International Journal of Computer Technology and Applications, Vol 3(3), 1049-1054, 2012.

[3]. H.K.Sawant, Mahendra Deore, " A Comprehensive Review of Image Enhancement Techniques", International Journal of Computer Technology and Electronics Engineering, Vol. 1, Issue 2, 2012.

[4]. Raju Rajkumar, K.Hemachandran,"A Review on Image Enhancement of Fingerprint using Directional Filters", Assam University Journal of Science and Technology, Vol. 7,52-57,2011.

[5]. Hartwig Fronthaler, Klaus Kollreider, Jodef Bigun, "Local Features for Enhancement and Minutiae Extraction in Fingerprints", Vol.17, 2008.

[6]. Mehdi Keshavarz, Javad Mohammedali, Rohollah Akbari, " Fingerprint Image Enhancement Using GWT and DMF", IEEE, 2010. 
[7]. Iwasokun G et al, "Multi-Level Model for fingerprint Image Enhancement", IMECS 2012, March 14-16, 2012, Hong Kong.

[8]. Shlomo Greenberg, Mayer Aledjem and Daniel Kogan, "Fingerprint Image Enhancement Techniques", Real Time Imaging, Elsevier 2002.
[9]. Sugata Ghosal et al., "Learning Partitioned Least Squares Filters for Fingerprint Enhancement", IEEE 2000.

[10].S.Arivazaghan and L.Ganesan, " Fingerprint Verifivation using Gabor Co-occurence Features”, ICCIM-2007. 